\title{
ON THE SIMULTANEOUS APPROXIMATION OF A FUNCTION AND ITS DERIVATIVES BY SUMS OF BIRKHOFF TYPE*
}

W. H. MCEWEN

\section{Introduction. Let}

$$
\begin{aligned}
L(u)+\lambda u \equiv u^{(n)}+P_{2}(x) u^{(n-2)} & +\cdots+P_{n}(x) u+\lambda u=0, \\
W_{j}(u) & =0, \quad j=1,2, \cdots, n,
\end{aligned}
$$

be a given linear differential system of the $n$th order subject to the following hypotheses:

(i) the functions $P_{2}, \cdots, P_{n}$ are continuous and have continuous derivatives of all orders on $(0,1)$;

(ii) the boundary conditions, consisting of $n$ linearly independent linear equations involving $u^{(k)}(0), u^{(k)}(1),(k=0,1, \cdots, n-1)$, are regular; $\dagger$

(iii) $\lambda=0$ is not a characteristic value, so that the system $L(u)=0$, $W_{j}(u)=0$ is incompatible.

Under hypotheses (i), (ii), it is well known that (1) possesses an infinite sequence of characteristic values $\left\{\lambda_{i}\right\}$ (arranged in order of increasing moduli) and a corresponding sequence of characteristic solutions $\left\{u_{i}(x)\right\}$. Moreover, the values $\lambda_{i}$ are also the poles of the Green's function $G(x, y ; \lambda)$ associated with (1), and these poles are, in general, simple when $\left|\lambda_{i}\right|$ is large. $\ddagger$ Furthermore, the system $L^{\prime}(v)+\lambda v=0, W_{j}^{\prime}(v)=0$, which is adjoint to (1), has the same characteristic values as (1), and a corresponding sequence of characteristic solutions $\left\{v_{i}(x)\right\}$.

For a given function $f(x)$, the Birkhoff series associated with (1) is defined by

$$
\sum_{i=1}^{\infty} \frac{\int_{0}^{1} f(y) v_{i}(y) d y}{\int_{0}^{1} u_{i}(y) v_{i}(y) d y} \cdot u_{i}(x),
$$

provided the poles of $G(x, y ; \lambda)$ are simple. In the case of multiple poles $\lambda_{\alpha}$, the corresponding terms in (2) are to be replaced by the terms $\int_{0}^{1} f(y) R_{\alpha}(x, y) d y$, where $R_{\alpha}(x, y)$ is the residue of $G$ at $\lambda=\lambda_{\alpha}$.

* Presented to the Society, December 30, 1937.

$\dagger$ For a definition of this term see G. D. Birkhoff, Transactions of this Society, vol. 9 (1908), pp. 373-395; p. 382.

$¥$ This is always so in the case when $n$ is odd, or when $n$ is even and the system is self-adjoint. When $n$ is even and the system is not self-adjoint, there may be an infinite number of double poles. 
The partial sums of this series will be denoted hereafter by $B_{N}(x)$. On the other hand, any arbitrary linear combination of the form

$$
S_{N}(x)=\sum_{i=1}^{N} c_{i} u_{i}(x)
$$

will be called "an arbitrary Birkhoff sum" or "a sum of Birkhoff type."

In this paper we propose to consider the possibility of approximating simultaneously a given function $f(x)$ and its first $m$ derivatives by sums of Birkhoff type, in the form

$$
\left|f^{(k)}(x)-S_{N}^{(k)}(x)\right| \leqq \epsilon_{N}
$$

uniformly on $(0,1)$, for $k=0,1, \cdots, m$, with $\lim _{N \rightarrow \infty} \epsilon_{N}=0$. The results obtained will then be used to derive certain theorems relating to the corresponding approximations when the sums $B_{N}(x)$ themselves are used. In this latter connection, we should mention the results given by Stone* relative to the derived series of Birkhoff, which, however, are different from those developed here.

2. Approximations using $S_{N}(x)$, when $m=n-1$. Let

$$
\sigma_{N}(x)=\sum_{i=1}^{N} a_{i} u_{i}(x)
$$

be an arbitrary Birkhoff sum of the $N$ th order, and consider the sum

$$
S_{N}(x)=\sum_{i=1}^{N} \frac{a_{i}}{\left(-\lambda_{i}\right)} u_{i}(x) .
$$

Since $u_{i}$ satisfies (1) when $\lambda=\lambda_{i}$, it follows that

$$
\begin{aligned}
L\left(S_{N}\right) & =\sum_{i=1}^{N} \frac{a_{i}}{\left(-\lambda_{i}\right)} L\left(u_{i}\right)=\sum_{i=1}^{N} a_{i} u_{i}=\sigma_{N}, \\
W_{j}\left(S_{N}\right) & =\sum_{i=1}^{N} \frac{a_{i}}{\left(-\lambda_{i}\right)} W_{j}\left(u_{i}\right)=0, \quad j=1,2, \cdots, n .
\end{aligned}
$$

That is, $S_{N}$ satisfies the nonhomogeneous system $L(y)=\sigma_{N}, W_{j}(y)=0$. But this system, in view of hypothesis (iii), has a unique solution, and hence, if $G(x, \xi)$ is the Green's function associated with $L(y)=0$, $W_{j}(y)=0$ (not to be confused with $G(x, y ; \lambda)$ ), we can write

$$
S_{N}^{(k)}(x)=\int_{0}^{1} \sigma_{N}(\xi) \frac{\partial^{k}}{\partial x^{k}} G(x, \xi) d \xi, \quad 0 \leqq x \leqq 1,
$$
761.

* M. H. Stone, Transactions of this Society, vol. 28 (1926), pp. 695-761; pp. 740- 
for $k=0,1, \cdots, n-1$. The functions $\partial^{k} G / \partial x^{k}$ in this expression exist and are known to be uniformly bounded on $0 \leqq x, \xi \leqq 1$ for all values of $k$ in question.

In regard to the function $f(x)$, let us assume that it satisfies the $n$ auxiliary equations $W_{j}(f)=0,(j=1,2, \cdots, n)$. This assumption is necessary if the errors of approximation in question are to converge to zero uniformly on $(0,1){ }^{*}$ With this understanding, the function $f(x)$ is the unique solution of the nonhomogeneous system $L(y)=L(f)$, $W_{j}(y)=0$, and hence

$$
f^{(k)}(x)=\int_{0}^{1} L(f)_{\xi} \frac{\partial^{k}}{\partial x^{k}} G(x, \xi) d \xi, \quad k=0,1, \cdots, n-1 .
$$

The functions $\partial^{k} G / \partial x^{k}$ being uniformly bounded on $0 \leqq x, \xi \leqq 1$ for all values of $k$ concerned, in the form $\left|\partial^{k} G / \partial x^{k}\right| \leqq \bar{G}$, it follows, from (3) and (4), that

$$
\begin{aligned}
\left|f^{(k)}-S_{N}^{(k)}\right| & =\left|\int_{0}^{1}\left(L(f)-\sigma_{N}\right) \frac{\partial^{k} G}{\partial x^{k}} d \xi\right| \\
& \leqq \bar{G} \int_{0}^{1}\left|L(f)-\sigma_{N}\right| d \xi, \quad 0 \leqq x \leqq 1,
\end{aligned}
$$

for $k=0,1, \cdots, n-1$.

From (5) it is clear that the errors $\left|f^{(k)}-S_{N}(k)\right|$ converge to zero uniformly on $(0,1)$, for $k=0,1, \cdots, n-1$, provided the sums $\sigma_{N}$ converge in the mean (with index 1) to the function $L(f)$. But the general theory of convergence in the mean tells us that such sums $\sigma_{N}$ do exist, provided $L(f)$ is integrable on $(0,1)$. For, in that case, it is possible to define an absolutely continuous function $\phi$ to satisfy $\int_{0}^{1}|L(f)-\phi| d x<\epsilon$ where $\epsilon>0$ is arbitrary. $\dagger$ The function $\phi$ belongs to the Lebesgue class $L_{2}$, and hence there must exist sums $\sigma_{N}$ which converge in the mean to $\phi$ with index $2, \ddagger$ and, therefore, also with index 1 by virtue of Schwarz's inequality. These facts, together with the inequality

* If the equations $W_{j}(f)=0$ are not satisfied, it can be shown that the sums $S_{N}^{(k)}$ converge to $f^{(k)}-W_{1}(f) G_{1}^{(k)}(x)-\cdots-W_{n}(f) G_{n}^{(k)}(x)$ rather than to $f^{(k)}$, where $G_{i}(x)$ is the solution of $L(y)=0, W_{j}(y)=0,(j \neq i), W_{i}(y)=1$.

$\dagger$ See, for example, E. C. Titchmarsh, The Theory of Functions, Oxford, 1932, p. 376.

$\ddagger$ As sums $\sigma_{N}$ we may choose the partial sums of the Birkhoff series for $L(f)$. These sums converge in the mean (with index 2) under the same hypotheses as will insure convergence in the mean of the Fourier series. This fact follows as an immediate consequence of Theorem XIII of Stone's paper, loc. cit., p. 723, and Minkowski's identity. 


$$
\int_{0}^{1}\left|L(f)-\sigma_{N}\right| d x \leqq \int_{0}^{1}|L(f)-\phi| d x+\int_{0}^{1}\left|\phi-\sigma_{N}\right| d x,
$$

prove the truth of the assertion. The condition that $L(f)$ be integrable is equivalent, of course, to the requirement that $f(x)$ and its first $n-1$ derivatives be continuous and $f^{(n)}(x)$ be integrable. Thus we have proved the following result.

THEOREM A. If $f(x)$ satisfies the auxiliary equations $W_{j}(f)=0$, $(j=1,2, \cdots, n)$, and $L(f)$ is integrable, then for every $\epsilon>0$ there exists a sum $S_{N}(x)$ of Birkhoff type such that $\left|f^{(k)}(x)-S_{N}^{(k)}(x)\right|<\epsilon$ uniformly on $(0,1)$ for $k=0,1, \cdots, n-1$.

3. The general case, when $m=p n-1$. We shall next show how the results of $\$ 2$ may be extended to the case $m=2 n-1$. It will then be obvious that the results may be extended to the general case $m=$ $p n-1$, where $p$ is any positive integer.

Let $\sigma_{N}$ be defined as in $\S 1$, and let $S_{N}(x)$ be redefined by the equation

$$
S_{N}(x)=\sum_{i=1}^{N} \frac{a_{i}}{\left(-\lambda_{i}\right)^{2}} u_{i}(x) .
$$

Also let $\tau_{N}$ denote the sum

$$
\sum_{i=1}^{N} \frac{a_{i}}{\left(-\lambda_{i}\right)} u_{i}(x)
$$

Then, since $L\left(\tau_{N}\right)=\sigma_{N}$ and $L\left(S_{N}\right)=\tau_{N}$, it is clear that

$$
\tau_{N}^{(k)}=\int_{0}^{1} \sigma_{N}(\xi) \frac{\partial^{k}}{\partial x^{k}} G(x, \xi) d \xi, \quad S_{N}^{(k)}=\int_{0}^{1} \tau_{N}(\xi) \frac{\partial^{k}}{\partial x^{k}} G(x, \xi) d \xi
$$

for $k=0,1, \cdots, n-1$.

In regard to the function $f(x)$, we must now assume that it satisfies the $2 n$ auxiliary conditions

$$
W_{j}(f)=0, \quad W_{j}(L(f))=0, \quad j=1,2, \cdots, n .
$$

Then, letting $F(x)$ denote the function $L(f)$, we can write

$$
F^{(k)}=\int_{0}^{1} L(F)_{\xi} \frac{\partial^{k}}{\partial x^{k}} G(x, \xi) d \xi, \quad f^{(k)}=\int_{0}^{1} F(\xi) \frac{\partial^{k}}{\partial x^{k}} G(x, \xi) d \xi .
$$

From these results and (6), it follows that 


$$
\begin{aligned}
& \left|f^{(k)}-S_{N}^{(k)}\right| \leqq \bar{G} \int_{0}^{1}\left|F-\tau_{N}\right| d \xi, \\
& \left|F^{(k)}-\tau_{N}^{(k)}\right| \leqq \bar{G} \int_{0}^{1}\left|L(F)-\sigma_{N}\right| d \xi .
\end{aligned}
$$

On putting $k=0$ in (9) and substituting the result in (8), we get

$$
\left|f^{(k)}-S_{N}^{(k)}\right| \leqq \bar{G}^{2} \int_{0}^{1}\left|L(F)-\sigma_{N}\right| d \xi, k=0,1, \cdots, n-1 .
$$

Again, from the identity

$$
f^{(n)}-S_{N}^{(n)} \equiv\left(F-\tau_{N}\right)-P_{2}\left(f^{(n-2)}-S_{N}^{(n-2)}\right)-\cdots-P_{n}\left(f-S_{N}\right),
$$

we obtain, with the help of (9) and (10),

$$
\left|f^{(n)}-S_{N}^{(n)}\right| \leqq K_{2} \int_{0}^{1}\left|L(F)-\sigma_{N}\right| d \xi .
$$

Likewise, after differentiating successively in (11), we can show that the errors $\left|f^{(n+1)}-S_{N}^{(n+1)}\right|, \cdots,\left|f^{(2 n-1)}-S_{N}^{(2 n-1)}\right|$ are similarly bounded. Since $F \equiv L(f)$, it will be convenient to denote $L(F)$ by $L^{2}(f)$. Hence, what we have proved may be restated as follows:

$$
\left|f^{(k)}-S_{N}^{(k)}\right| \leqq K^{\prime} \int_{0}^{1}\left|L^{2}(f)-\sigma_{N}\right| d \xi, \quad 0 \leqq x \leqq 1,
$$

for $k=0,1, \cdots, 2 n-1$.

In the general case, when $m=p n-1$, we define $S_{N}(x)$ by the equation

$$
S_{N}(x)=\sum_{i=1}^{N} \frac{a_{i}}{\left(-\lambda_{i}\right)^{p}} u_{i}(x) .
$$

We must now assume that $f(x)$ satisfies the $p n$ auxiliary conditions

$$
W_{j}(f)=0, W_{j}(L(f))=0, \cdots, W_{j}\left(L^{p-1}(f)\right)=0, \quad j=1,2, \cdots, n .
$$

Then it may be shown that

$$
\left|f^{(k)}-S_{N}^{(k)}\right| \leqq K \int_{0}^{1}\left|L^{p}(f)-\sigma_{N}\right| d \xi
$$

uniformly on $(0,1)$ for $k=0,1, \cdots, p n-1$.

Thus we can state the following generalization of Theorem A. 
THEOREM 1. If $f(x)$ satisfies the pn auxiliary equations (12) and $L^{p}(f)$ is integrable, then for every $\epsilon>0$ there exists a sum $S_{N}(x)$ of Birkhoff type such that $\left|f^{(k)}(x)-S_{N}{ }^{(k)}(x)\right|<\epsilon$ uniformly on $(0,1)$ for $k=0,1, \cdots, p n-1$.

4. Approximations using $B_{N}(x)$. In this section we shall deal only with the case when $m=n-1$, although the results obtained hold generally when $m=p n-1$.

Let $\sigma_{N}$ be identified with the partial sum of the Birkhoff series for $L(f)$. Then, if $\lambda_{i}$ is a simple pole of $G(x, y ; \lambda)$,

$$
a_{i}=\frac{\int_{0}^{1} L(f) v_{i} d y}{\int_{0}^{1} u_{i} v_{i} d y}
$$

From Green's identity and the fact that $W_{j}(f)=0,(j=1,2, \cdots, n)$, we have

$$
\int_{0}^{1}\left[v_{i} L(f)-f L^{\prime}\left(v_{i}\right)\right] d y=0 .
$$

But $v_{i}$ satisfies the adjoint system when $\lambda=\lambda_{i}$, so that $L^{\prime}\left(v_{i}\right)+\lambda_{i} v_{i}=0$, and hence $\int_{0}^{1} v_{i} L(f) d y=\int_{0}^{1} f L^{\prime}\left(v_{i}\right) d y=-\lambda_{i} \int_{0}^{1} f v_{i} d y$. That is,

$$
\frac{a_{i}}{\left(-\lambda_{i}\right)}=\frac{\int_{0}^{1} f v_{i} d y}{\int_{0}^{1} u_{i} v_{i} d y}
$$

which means that $-a_{i} / \lambda_{i}$ is a coefficient of the partial sum of the Birkhoff series for $f(x)$.

On the other hand, if $\lambda_{i}$ is a multiple pole of $G(x, y ; \lambda)$, the corresponding terms $a_{i} u_{i}$ in $\sigma_{N}$ must be replaced by $\int_{0}^{1} L(f) R_{i}(x, y) d y$, where $R_{i}(x, y)$ is the residue at $\lambda=\lambda_{i}$. But from the known properties of the residue, we can easily infer that

$$
\int_{0}^{1} L(f) R_{i}(x, y) d y /\left(-\lambda_{i}\right)=\int_{0}^{1} f R_{i}(x, y) d y .
$$

It follows, then, that if $S_{N}(x)$ is defined by

$$
S_{N}(x)=\sum_{i=1}^{N} \frac{a_{i}}{\left(-\lambda_{i}\right)} u_{i}(x),
$$

with the understanding that the terms $-a_{i} u_{i} / \lambda_{i}$ are to be replaced by $\int_{0}^{1} L(f) R_{i}(x, y) d y /\left(-\lambda_{i}\right)$ in the case of multiple poles, the sum $S_{N}(x)$ is identical with $B_{N}(x)$, the partial sum of the Birkhoff series for $f(x)$. The discussion given in $\S 2$, with appropriate modifications for the case 
of multiple poles, will then apply to $B_{N}(x)$ to show that the errors $\left|f^{(k)}-B_{N}{ }^{(k)}\right|$ converge to zero uniformly on $(0,1)$ for $k=0,1, \cdots$, $n-1$, provided the partial sums of the Birkhoff series for $L(f)$ converge in the mean (with index 1 ) to $L(f)$ as $N \rightarrow \infty$. But, as we have already noted in the third footnote on p. 578 , this latter convergence is assured if $L(f)$ is integrable.

There is no difficulty in extending these results to the case $m=p n-1$. Hence we can state our next theorem.

THEOREM 2. If $f(x)$ satisfies the pn auxiliary equations (12) and $L^{p}(f)$ is integrable, then the partial sums of the Birkhoff series for $f(x)$ are such that $\lim _{N \rightarrow \infty} B_{N}^{(k)}(x)=f^{(k)}(x)$ uniformly on $(0,1)$, for $k=0,1, \cdots, p n-1$.

The inequalities (13) enable us to write

$$
\left|f^{(k)}-S_{N}^{(k)}\right| \leqq K \max \left|L^{p}(f)-\sigma_{N}\right|
$$

uniformly on $(0,1)$, for $k=0,1, \cdots, p n-1$. But $f^{(p n)}-S_{N}^{(p n)}$ can be expressed by an identity analogous to (11), in the form $f^{(p n)}-S_{N}(p n) \equiv\left(L^{p}(f)-\sigma_{N}\right)+\cdots$, in which the terms on the right are all uniformly bounded by constant multiples of $\max \left|L^{p}(f)-\sigma_{N}\right|$. Hence, there must exist a constant $\bar{K}$ such that

$$
\left|f^{(k)}-S_{N}^{(k)}\right| \leqq \bar{K} \max \left|L^{p}(f)-\sigma_{N}\right|
$$

uniformly on $(0,1)$, for $k=0,1, \cdots, p n$. This inequality, with the help of Milne's results relating to the degree of approximation of the Birkhoff series, ${ }^{*}$ enables us to state the following theorem.

THEOREM 3. If $f(x)$ satisfies the pn equations (12) and $L^{p}(f)$ and its first $q-1$ derivatives vanish at 0 and 1 , its qth derivative being continuous and of limited variation on $(0,1)$, then the partial sums of the Birkhoff series for $f(x)$ are such that $\left|f^{(k)}-B_{N}{ }^{(k)}\right|<K / N^{q}$ uniformly on $(0,1)$, for $k=0,1, \cdots, p n$, where $K$ is a constant independent of $N$.

\section{Mount Allison University}

* W. E. Milne, Transactions of this Society, vol. 19 (1918), pp. 143-156, Theorem I. 